\title{
Tuning the Shape and Catalytic Activity of Fe Nanocrystals from Rhombic Dodecahedra and Tetragonal Bipyramids to Cubes by Electrochemistry
}

\author{
Yan-Xin Chen, ${ }^{\dagger}$ Sheng-Pei Chen, ${ }^{\dagger}$ Zhi-You Zhou, ${ }^{\dagger}$ Na Tian, ${ }^{\dagger}$ Yan-Xia Jiang, ${ }^{\dagger}$ Shi-Gang Sun, ${ }^{*},{ }^{\dagger}$ \\ Yong Ding, ${ }^{\ddagger}$ and Zhong Lin Wang ${ }^{\ddagger}$ \\ State Key Laboratory of Physical Chemistry of Solid Surfaces, Department of Chemistry, College of Chemistry and \\ Chemical Engineering, Xiamen University, Xiamen 361005, China, and School of Materials Science and \\ Engineering, Georgia Institute of Technology, Atlanta, Georgia 30332-0245
}

Received May 25, 2009; E-mail: sgsun@xmu.edu.cn

The catalytic properties of nanocrystals (NCs) can be precisely tuned either by modifying their composition, which mediates the electronic structure,${ }^{1}$ or by tailoring their shape, which determines the surface atomic arrangement and coordination. ${ }^{2}$ Therefore, the shape-controlled synthesis of nanocrystals presents an important direction for tuning the activity, stability, and selectivity of nanocrystal catalysts. Although a variety of well-defined shapes of metal NCs with enhanced optical, electronic, and catalytic properties have been synthesized in the past decade, most of them are bounded by closest-packing facets because of the limit of crystal growth rule, which necessitates a minimization of the surface energy of the NCs. ${ }^{3}$ As the closest-atomic-packed facets are composed of atoms with high coordination number and accordingly have a low surface energy, they are stable but in general exhibit low catalytic activities for chemical reactions. ${ }^{4,5}$ The synthesis of NCs bounded by crystalline facets with an open structure, i.e., with surface atoms of low coordination number and thus high surface energy, presents a promising avenue in catalyst design and synthesis, although it is highly challenging. ${ }^{6}$

$\mathrm{Fe}$ is the fourth most abundant element on earth. Fe nanoparticles have been extensively investigated because of their wide application. ${ }^{7} \mathrm{Fe}$ NCs are very important catalysts in ammonia synthesis. Somorjai and co-workers ${ }^{8}$ have demonstrated that the surface structures of $\mathrm{Fe}$ catalysts have a significant impact on their reactivity. The $\mathrm{Fe}(111)$ plane (an open-structure surface for the bcc lattice) is the most active, while the $\mathrm{Fe}(110)$ plane (the closestpacking surface) is the most inactive. They determined that for $\mathrm{Fe}$ single-crystal planes, the $(111) /(100) /(110)$ reactivity ratio is 418 : $25: 1{ }^{8 \mathrm{a}} \mathrm{Fe}$ also plays a very important role in denitrification, which is very important in today's society since the intensive use of fertilizers in agriculture and nitrates in some industries causes severe nitrate/nitrite pollution of water sources.

Recently, extensive efforts to synthesize Fe nanoparticles for different applications have been made. Ling et al. ${ }^{9}$ reported the synthesis of icosahedral face-centered cubic (fcc) Fe nanoparticles with sizes of 5-13 $\mathrm{nm}$ through a specifically designed thermodynamically governed synthetic route. We have synthesized Fe cuboid nanoparticles supported on glassy carbon by electrochemical deposition under cyclic voltammetric conditions. ${ }^{10}$

In the present work, two series of Fe NCs enclosed by different crystalline facets were synthesized by means of an electrochemical route. We demonstrated that the shape of the Fe NCs can be finely tuned systematically by varying the electrochemical conditions, i.e., the deposition potential and the concentration of $\mathrm{FeSO}_{4}$ in solution. The Fe NCs were tuned from rhombic dodecahedra or tetragonal

Xiamen University.

* Georgia Institute of Technology. bipyramids bounded by $\{110\}$ facets to a series of 18 -facet polyhedra enclosed by different combinations of $\{110\}$ and $\{100\}$ facets and finally to cubes of $\{100\}$ facets. With the synthesized $\mathrm{Fe} \mathrm{NCs,} \mathrm{the} \mathrm{surface-structure} \mathrm{functionality} \mathrm{of} \mathrm{the} \mathrm{Fe} \mathrm{NCs} \mathrm{toward}$ electrocatalytic reduction of nitrite was investigated.

Fe NCs were synthesized by electrodeposition on a glassy carbon (GC) substrate. It is known that the surface energy of GC is very low, allowing metal nanoparticles to preferentially grow to individual particles or clusters through the Volmer-Weber mode. ${ }^{11}$ This favors the shape-controlled synthesis of metal nanocrystals. ${ }^{6}$

In a typical synthesis process (Figure S1 in the Supporting Information), $\mathrm{Fe}$ nanoseeds with sizes of $3-8 \mathrm{~nm}$ were first nucleated on the GC electrode surface at a nucleation potential $\left(E_{\text {nuc }}\right)$ for several seconds $\left(t_{\text {nuc }}\right)$ in $0.1 \mathrm{M} \mathrm{Na}_{2} \mathrm{SO}_{4}$ solutions containing different concentrations of $\mathrm{FeSO}_{4}$. The $\mathrm{Fe}$ nanoseeds were then grown slowly at a relatively positive potential $\left(E_{\text {growth }}\right)$ for a defined period of time $\left(t_{\text {growth }}\right)$. The shape of the Fe NCs could be tuned precisely by careful control of the electrodeposition parameters, i.e., $E_{\text {nuc }}, t_{\text {nuc }}, E_{\text {growth }}, t_{\text {growth }}$, and the $\mathrm{FeSO}_{4}$ concentration (the experimental details and corresponding $\mathrm{Fe} \mathrm{NC}$ shapes are illustrated in Figure S2). The electrode of Fe NCs deposited on GC (hereafter denoted nano-Fe/GC) was then taken out of the solution at the same potential of $E_{\text {growth }}$ and immediately put into absolute alcohol to protect the Fe NCs from oxidation before subsequent characterizations. The morphology of the Fe NCs on the GC substrate was examined by scanning electron microscopy (SEM). X-ray diffraction (XRD) and X-ray photoelectron spectroscopy (XPS) confirmed that the as-synthesized NCs consisted of Fe with a bcc lattice (Figure S3).

Figure 1a shows an SEM image of rhombic-dodecahedral (RD) Fe NCs obtained in $0.002 \mathrm{M} \mathrm{FeSO}_{4}+0.1 \mathrm{M} \mathrm{Na}_{2} \mathrm{SO}_{4}(\mathrm{pH} 3-3.5)$ with $E_{\text {nuc }}, t_{\text {nuc }}, E_{\text {growth }}$, and $t_{\text {growth }}$ at $-1.2 \mathrm{~V}, 4.02 \mathrm{~s},-1.03 \mathrm{~V}$, and $900 \mathrm{~s}$, respectively. It can be seen that the Fe NCs are fairly uniform in shape and size but their orientations on the GC surface are random. High-magnification SEM images of RD Fe NCs oriented nearly along the [110] and [100] axes and corresponding model illustrations are presented in Figure $1 \mathrm{~b}-\mathrm{e}$ (Figure S4 shows the [111] orientation). The yield of the RD Fe NCs in the final product was $>78 \%$, and their average size was $132 \mathrm{~nm}$ with a standard deviation of $32 \mathrm{~nm}$ (Figure S4).

The RD shape of the Fe NCs was further confirmed by selectedarea electron diffraction (SAED) and transmission electron microscopy (TEM). The SAED pattern along the [011] axis confirmed that the RD Fe NC is a single crystal (Figure 1f). The facets were best revealed by imaging the $\mathrm{NC}$ along the [011] axis, as shown in Figure $1 \mathrm{~g}$, in which the projection lines of two parallel facets and four edges constitute a hexagon. The angle $\alpha$ between the projection lines of facet and edge was measured to be $125.3 \pm 0.8^{\circ}$, and the 


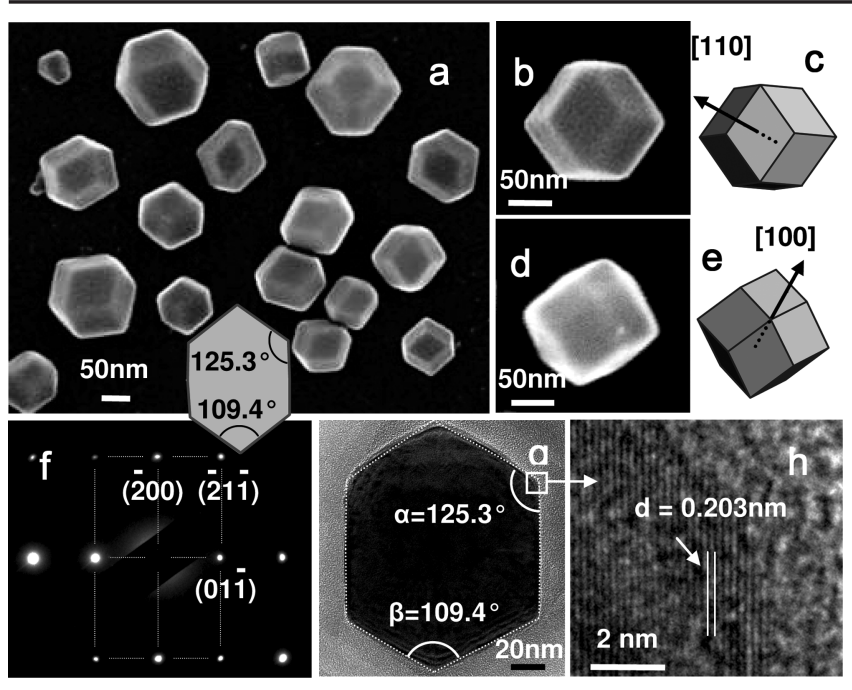

Figure 1. (a) SEM image of $\mathrm{Fe} \mathrm{NCs}$ obtained in $0.002 \mathrm{M} \mathrm{FeSO}_{4}+0.1 \mathrm{M}$ $\mathrm{Na}_{2} \mathrm{SO}_{4}$ (pH 3-3.5). (b-e) Two typical high-magnification SEM images and corresponding geometrical models of RD Fe NCs oriented nearly along the [110] and [100] axes. (f) SAED pattern of an RD Fe NC along the [011] axis. (g) TEM image of an RD Fe NC recorded along the [011] direction. (h) HRTEM image recorded from the boxed area marked in $(\mathrm{g})$.

angle $\beta$ between the projection lines of two edges was $109.4 \pm$ $0.6^{\circ}$; both are in good agreement with the theoretical model illustrated in the inset to this figure. The corresponding highresolution TEM (HRTEM) image along the [011] axis is shown in Figure 1h, from which the crystal lattice spacing was measured as $0.203 \mathrm{~nm}$, in good accord with the $\{110\}$ plane spacing of the iron bcc crystal structure. The SAED pattern and corresponding HRTEM image taken along the [111] axis (Figure $\mathrm{S} 4 \mathrm{e}-\mathrm{g}$ ) also verified this point. These results demonstrate that the dominant facets of the RD Fe NCs are $\{110\}$.

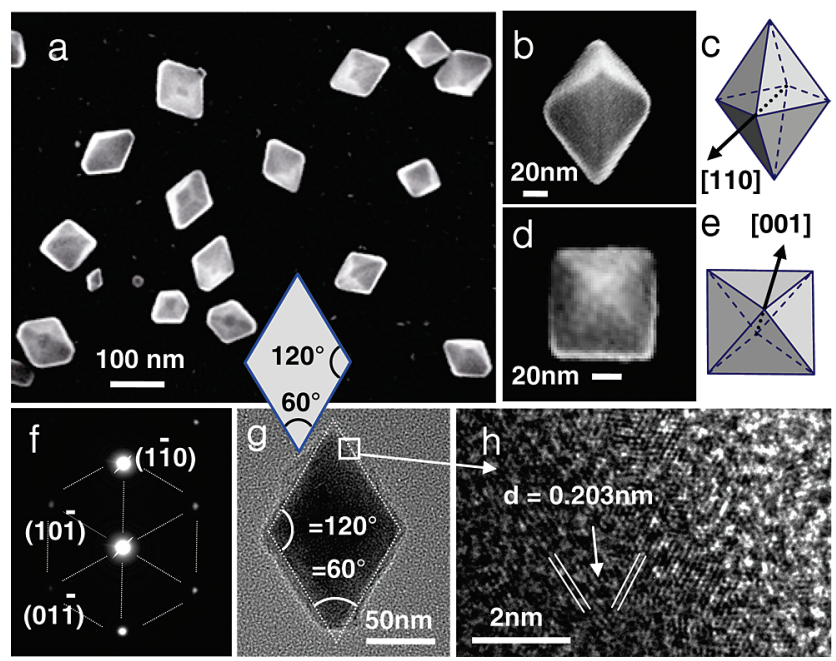

Figure 2. (a) SEM image of Fe NCs obtained in $0.02 \mathrm{M} \mathrm{FeSO}_{4}+0.1 \mathrm{M}$ $\mathrm{Na}_{2} \mathrm{SO}_{4}$ (pH 3-3.5). (b-e) Two typical high-magnification SEM images and corresponding geometrical models of TB Fe NCs oriented nearly along the [110] and [001] axes. (f) SAED pattern of a TB Fe NC along the [111] axis. (g) TEM image of a TB Fe NC recorded along the [111] direction. (h) HRTEM image recorded from the boxed area marked in $(\mathrm{g})$.

Usually, the shape of crystals enclosed by $\{110\}$ facets is RD for cubic crystals, as shown in Figure 1. Interestingly, however, when the concentration of $\mathrm{FeSO}_{4}$ was increased to $0.02 \mathrm{M}$, we obtained for the first time Fe NCs with a new shape, tetragonal bipyramidal (TB), enclosed by eight $\{110\}$ facets. Figure $2 \mathrm{a}$ is a SEM image of freshly prepared TB Fe NCs. Figure $2 b-e$ shows two typical high-magnification SEM images and corresponding models of TB Fe NCs oriented nearly along the [110] and [001] axes (TB Fe NCs oriented nearly along the [100] and [111] axes and the corresponding models are shown in Figure S5c-f), demonstrating clearly the well-defined TB shape. The yield of the TB Fe NCs in the final product was $>58 \%$, and their average size was measured to be $110 \pm 26 \mathrm{~nm}$ (Figure S5a,b).

Figure $2 \mathrm{f}, \mathrm{g}$ shows the SAED pattern and TEM image of a TB Fe NC along the [111] axis. It is interesting that the TEM image shows a rhombic projection. Such a rhombic projection excludes the possibility that the $\mathrm{Fe} \mathrm{NC}$ is regular octahedron, for which a square projection should be obtained. The SAED pattern confirmed that the TB Fe NC is a single crystal. As shown in Figure $2 \mathrm{~g}$, two angles of 120 and $60^{\circ}$ were measured, in good accord with theoretical values of the angles between two $\{110\}$ facets (see the inset model). The results indicate that the dominant facets of the TB Fe NCs are $\{110\}$, which was also verified by the SAED and TEM results along the [110] axis (Figure S5). The $\mathrm{FeSO}_{4}$ concentration in solution is certainly the main factor that affects the development of the Fe NCs enclosed with $\{110\}$ facets. On the RD Fe NCs, the $12\{110\}$ facets may develop slowly and isotropically at a low $\mathrm{FeSO}_{4}$ concentration, while at a high $\mathrm{FeSO}_{4}$ concentration, four $\{110\}$ facets may grow much faster than the remaining eight, leading to the formation of the TB Fe NCs. The fact that $\mathrm{Fe} \mathrm{NCs}$ enclosed by $\{110\}$ facets but having shapes between $\mathrm{RD}$ and $\mathrm{TB}$ were obtained at medium $\mathrm{FeSO}_{4}$ concentrations (Figure S6) further confirmed this aspect.

The shape of Fe NCs can be finely tuned by manipulating the electrodeposition potential. In $0.002 \mathrm{M} \mathrm{FeSO}_{4}+0.1 \mathrm{M} \mathrm{Na}_{2} \mathrm{SO}_{4}$, a transformation of the Fe NC shape from RD to a series of 18 -facet polyhedral shapes and finally to cubic was achieved when $E_{\text {growth }}$ was negatively shifted from -1.03 to $-1.07 \mathrm{~V}$ (Figure S2). Correspondingly, the surface structure of the Fe NCs was changed from $\{110\}$ to a certain combination of $\{110\}$ and $\{100\}$ and finally to $\{100\}$ exclusively (Figure $3 \mathrm{a}-\mathrm{e}$ ).
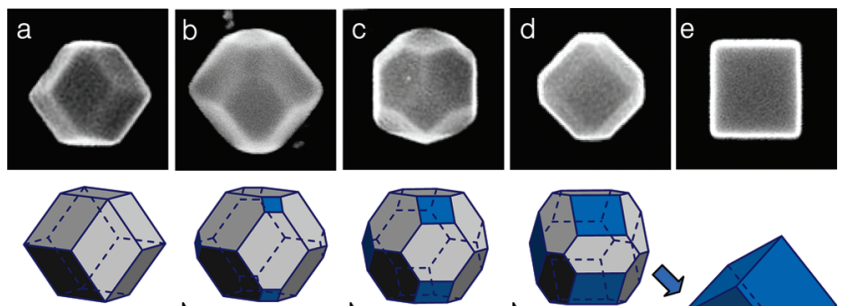

$\longrightarrow$
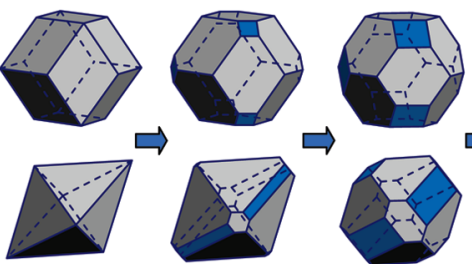

$\Rightarrow$

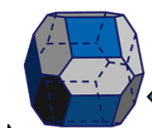

$\rightarrow$
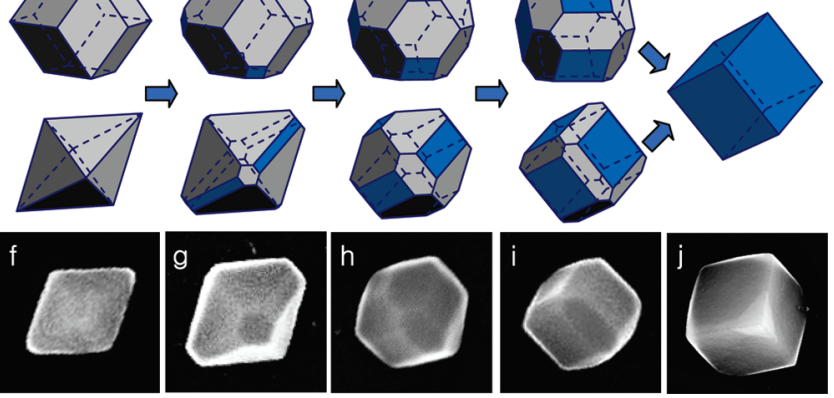

Figure 3. SEM images of Fe NCs and corresponding models. (a-e) Shape transformation of $\mathrm{Fe} \mathrm{NCs}$ from RD to a series of 18 -facet polyhedral shapes and finally to cubic in $0.002 \mathrm{M} \mathrm{FeSO}_{4}+0.1 \mathrm{M} \mathrm{Na}_{2} \mathrm{SO}_{4}(\mathrm{pH} 3-3.5)$. (f-j) Shape transformation of Fe NCs from TB to a series of 18 -facet polyhedral shapes and finally to cubic in $0.02 \mathrm{M} \mathrm{FeSO}_{4}+0.1 \mathrm{M} \mathrm{Na}_{2} \mathrm{SO}_{4}(\mathrm{pH} 3-3.5)$.

However, in $0.02 \mathrm{M} \mathrm{FeSO}_{4}+0.1 \mathrm{M} \mathrm{Na}_{2} \mathrm{SO}_{4}$, a transformation of the Fe NC shape from TB to a series of 18-facet polyhedral shapes and finally to cubic was accomplished (Figure $3 \mathrm{f}-\mathrm{j}$ ) when 
$E_{\text {growth }}$ was negatively shifted from -0.97 to $-1.025 \mathrm{~V}$. It is obvious that the fraction of $\{100\}$ facets increases as $E_{\text {growth }}$ becomes more negative, i.e., at higher overpotentials. This result is in agreement with the two-dimensional nuclei theory, ${ }^{12}$ which indicates that the rate of formation of two-dimensional nuclei of the type $\{h k l\}$ is proportional to $\exp \left(-W_{h k l} / k_{\mathrm{B}} T\right)$ (where $W_{h k l}$ is the work of formation of the $\{h k l\}$ nuclei, $k_{\mathrm{B}}$ is Boltzman's constant, and $T$ is the absolute temperature) and predicts that $W_{110}$ has the lowest value at low overpotentials while $W_{100}$ becomes lowest at relatively high overpotentials.
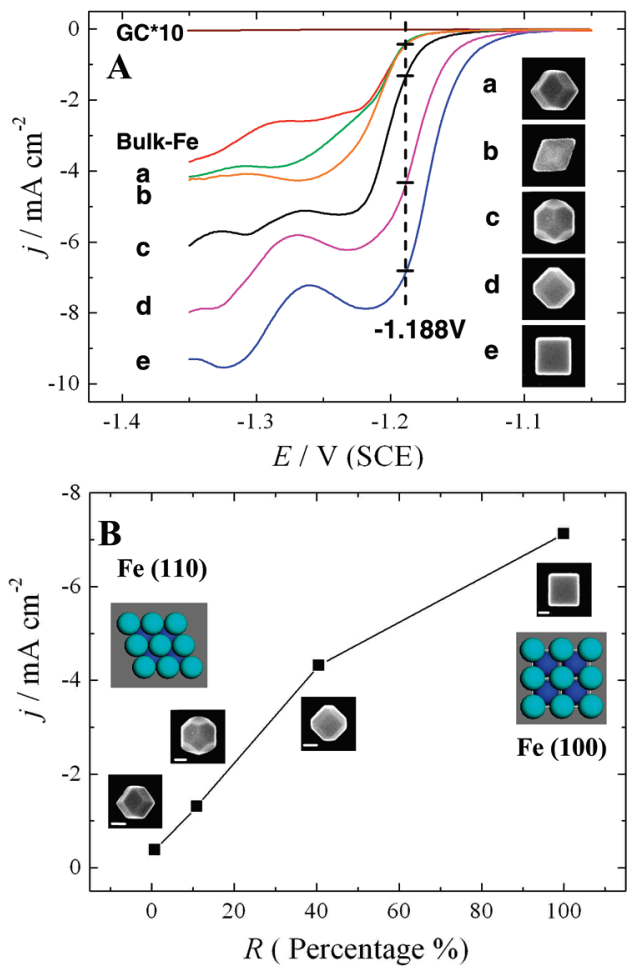

Figure 4. Electrocatalytic activity of Fe NCs toward nitrite reduction. (A) $j-E$ curves for $\mathrm{Fe}$ NCs with different shapes recorded on nano-Fe/GC electrodes in $0.01 \mathrm{M} \mathrm{NaNO}_{2}+0.2 \mathrm{M} \mathrm{NaOH}$ at a scan rate of $1 \mathrm{mV} \mathrm{s}^{-1}$. (B) Relationship between electrocatalytic activity of the Fe NCs, represented by $j$ measured at $-1.188 \mathrm{~V}$ in (A), and the ratio of active surface atoms. The scale bar in all of the SEM images is $50 \mathrm{~nm}$.

The electrocatalytic activity of the synthesized Fe NCs was tested using nitrite reduction. The reduction current density $(j)$ in Figure 4 was normalized to the surface area of the nano-Fe/GC electrodes calibrated using a bulk Fe electrode (Figure S7) ${ }^{10}$ and used directly to compare the catalytic activities of different samples. It was confirmed that the structures of the Fe NCs remained unchanged during the electrocatalytic reaction (Figure S8).

We clearly observe that the nitrite reduction starts at about $-1.084 \mathrm{~V}\left(E_{\text {onset }}\right)$ on the cubic nano-Fe/GC electrode. The values of $E_{\text {onset }}$ measured on nano-Fe/GC electrodes of RD and TB Fe $\mathrm{NCs}$ are more negative and close to the $E_{\text {onset }}$ on the bulk $\mathrm{Fe}$ electrode $(-1.181 \mathrm{~V})$ (Table $\mathrm{S} 1)$. This result clearly indicates that the electrocatalytic activity of the cubic Fe NCs with an opensurface $\{100\}$ structure is much higher than that of the RD and TB Fe NCs enclosed with closest-packed $\{110\}$ facets. The relationship between the surface structure of Fe NCs and their electrocatalytic activity toward nitrite reduction was analyzed by plotting the $j$ value measured at a given electrode potential (e.g., at $-1.188 \mathrm{~V}$, as indicated in Figure 4A) against the ratio of active surface atoms on an Fe NC (i.e., $R=N_{\text {active }} / N_{\text {total }}$ ). $N_{\text {active }}$ is the sum of the numbers of $\{100\}$, edge, and corner atoms (Figure S9), and $N_{\text {total }}$ is the total number of surface atoms on the Fe NC. Figure 4B illustrates the relationship between $j$ and $R$ for a series of Fe NCs with shapes ranging from RD to cubic. It is obvious that $j$ increases with $R$, demonstrating that the electrocatalytic activity of the Fe NCs is enhanced by increasing the fraction of $\{100\}$ facets on the Fe NC surface. This structural dependence of Fe NC electrocatalytic activity was also confirmed by the steady catalytic activity, which was measured from the time-dependent current density of nitrite reduction at a fixed potential over a long reaction time $(-1.188 \mathrm{~V}$ and 200 s; see Figure S10 and Table S1).

In summary, we have revealed in the present work that the shape of Fe nanocrystals can be finely tuned by careful control of the electrochemical conditions during the synthesis process. Transformations of $\mathrm{Fe} \mathrm{NCs}$ from rhombic dodecahedra or tetragonal bipyramids bounded by $\{110\}$ facets to 18 -facet polyhedra enclosed by certain combinations of $\{110\}$ and $\{100\}$ facets and finally to cubes exclusively covered by $\{100\}$ facets were successfully achieved. This study has revealed that the electrocatalytic activity of Fe NCs for nitrite reduction increases as the fraction of $\{100\}$ facets increases and that the cubic Fe NCs with high-energy $\{100\}$ surfaces exhibit the highest activity among the Fe NCs synthesized. This study demonstrates that the electrochemistry route is important and convenient for tailoring the electrocatalytic properties of metal nanoparticles by controlling their shape and surface structure and that the Fe NCs with an open surface structure may serve as efficient catalysts in important fields such as ammonia synthesis and denitrification.

Acknowledgment. This study was supported by the NSFC (20833005, 20873113, 20873116), the MOST (2007DFA40890), and the Fujian Key Project (2008I0025).

Supporting Information Available: Detailed synthesis and characterization procedures. This material is available free of charge via the Internet at http://pubs.acs.org.

\section{References}

(1) (a) Zhang, J.; Sasaki, K.; Sutter, E.; Adzic, R. R. Science 2007, 315, 220. (b) Stamenkovic, V. R.; Fowler, B.; Mun, B. S.; Wang, G. F.; Ross, P. N.; Lucas, C. A.; Markovic, N. M. Science 2007, 315, 493.

(2) (a) Narayanan, R.; El-Sayed, M. A. Nano Lett. 2004, 4, 1343. (b) Lee, H.; Habas, S. E.; Kweskin, S.; Butcher, D.; Somorjai, G. A.; Yang, P. D. Angew. Chem., Int. Ed. 2006, 45, 7824.

(3) (a) Sun, Y. G.; Xia, Y. N. Science 2002, 298, 2176. (b) Tao, A. R.; Habas, S.; Yang, P. D. Small 2008, 4, 310.

(4) Tian, N.; Zhou, Z. Y.; Sun, S. G. J. Phys. Chem. C 2008, 112, 19801.

(5) Somorjai, G. A. Science 1985, 227, 902.

(6) (a) Tian, N.; Zhou, Z. Y.; Sun, S. G.; Ding, Y.; Wang, Z. L. Science 2007, 316, 732. (b) Zhou, Z. Y.; Tian, N.; Huang, Z. Z.; Chen, D. J.; Sun, S. G. Faraday Discuss. 2008, 140, 81. (c) Tian, N.; Zhou, Z. Y.; Sun, S. G. Chem. Commun. 2009, 1502. (d) Liao, H.-G.; Jiang, Y.-X.; Zhou, Z.-Y.; Chen, S.-P.; Sun, S.-G. Angew. Chem. 2008, 120, 9240.

(7) Huber, D. L. Small 2005, 5, 482.

(8) (a) Spencer, N. D.; Schoonmaker, R. C.; Somorjai, G. A. Nature 1981, 294, 643. (b) Somorjai, G. A.; Park, J. Y. Catal. Lett. 2007, 115, 87.

(9) Ling, T.; Xie, L.; Zhu, J.; Yu, H. M.; Ye, H. Q.; Yu, R.; Cheng, Z. Y.; Liu, L.; Liu, L.; Yang, G. W.; Cheng, Z. D.; Wang, Y. J.; Ma, X. L. Nano Lett. 2009, 9, 1572.

(10) Chen, Y. X.; Chen, S. P.; Chen, Q. S.; Zhou, Z. Y.; Sun, S. G. Electrochim. Acta 2008, 53, 6938.

(11) Penner, R. M. J. Phys. Chem. B 2002, 106, 3339.

(12) Pangarov, N. A. J. Electroanal. Chem. 1965, 9, 70.

JA904225Q 